Check for updates

Cite this: Chem. Sci., 2019, 10, 257

๑ All publication charges for this article have been paid for by the Royal Society of Chemistry

\section{Hybrid CuCoO-GO enables ultrasensitive detection of antibiotics with enhanced laser desorption/ionization at nano-interfaces $\uparrow$}

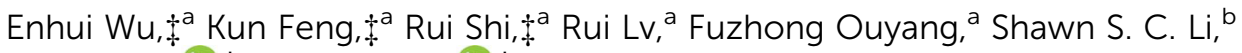 \\ Jun Zhong (D) *a and Jian Liu iD *a
}

\begin{abstract}
The soaring concerns globally on antibiotic overuse have made calls for the development of rapid and sensitive detection methods urgent. Here we report that the hybrid CuCoO-GO matrix allows for sensitive detection of various antibiotics in combination with MALDI TOF MS. The new matrix is composed of few-layered GO nanosheets decorated with $\mathrm{CuCoO}$ nanoparticles with an average size of $10 \mathrm{~nm}$, and exhibits excellent aqueous suspensibility. Accurate quantitation of the sulfonamide antibiotics in milk samples have been demonstrated using a $\mathrm{CuCoO}-\mathrm{GO}$ matrix and a stable isotope $\left(\mathrm{C}^{13}\right)$-labeled analyte as the internal standard. Our experiments have achieved lower limits of detection (LOD) by several hundred fold for the detection of a panel of representative antibiotics, in comparison with the literature reports. Both intrabacterial and extrabacterial residual antibiotics can be sensitively detected with our method. We have further investigated the molecular mechanism of the enhanced desorption/ ionization efficiency by the $\mathrm{CuCoO}-\mathrm{GO}$ matrix with synchrotron radiation techniques for the first time. This work provides a sensitive matrix enabling MALDI-TOF MS to be applied in small molecular analysis, but also presents a distinct perspective on the mechanism behind the material functions.
\end{abstract}

Received 18th August 2018

Accepted 5th October 2018

DOI: $10.1039 / c 8 s c 03692 f$

rsc.li/chemical-science
Chromatographic methods require lengthy procedures for sample-pretreatment. ${ }^{7,9}$ Receptor-based screening methods, such as radio-receptor assay, enzyme-labelling receptor assay, colloidal gold-labelling receptor assay and enzyme colorimetry are limited by enzymatic activity fluctuations and labellingreagent contamination..$^{12,13}$

Matrix-assisted laser desorption/ionization time-of-flight mass spectrometry (MALDI-TOF MS) is an indispensable tool for the analysis of biomolecules, with the advantages of high sensitivity, high throughput, and a fast procedure to obtain results. ${ }^{\mathbf{1 4 - 2 1}}$ However, the conventional organic matrices (CHCA, DHB, SA, etc.) widely used in MALDI-TOF MS are subject to their own structural fragmentation by lasers, which generates intensive background noise below $\mathrm{m} / \mathrm{z} 700$ intrinsically. This overwhelms the true signals of small molecular analytes, thus severely limiting the applications of MALDI-TOF MS in the $(\mathrm{m} / \mathrm{z}$ < 700) molecular range. ${ }^{22,23}$ Researchers have attempted to synthesize different types of organic molecules as replaceable matrix candidates to suppress background noise. ${ }^{24,25}$ Alternatively, various inorganic nanomaterials and nanostructures have been proposed as MALDI matrices to increase the signal to noise ratio in the detection of small molecules, including silicon, metal, metallic oxide and carbon-based nanomaterials. ${ }^{\text {26-34 }}$ Among them, graphene oxide (GO) is attractive due to its large surface area, good light absorption, and excellent optoelectric properties. ${ }^{32}$ It has been suggested that graphene oxide/multi-walled carbon nanotube composites can 
promote small molecule detection by MALDI MS because of the improved wettability in water and enlarged surface area. ${ }^{32}$ However, many nanomaterial-based matrices still suffer from drawbacks such as the background noise generated by their inherent structural defects, or the relatively lower efficiency in assisting molecular ionization. ${ }^{34-36}$

Herein, we report an important discovery and mechanism revelation for the sensitive detection of various antibiotics using hybrid CuCoO-GO as the MALDI matrix. Previously we reported that tuning the ratio of copper and cobalt atoms in their metal oxide-graphene composite could maximize the electron transfer properties for a synergistic catalytic performance in the hydrolysis of ammonia borane. ${ }^{37}$ In this work, we hypothesize that the electron transfer properties of hybrid CuCoO-GO could promote the ionization of small molecules under laser irradiation, with significant improvement in the signal to noise ratios in MS spectral analysis. We have demonstrated that the CuCoOGO matrix enables the detection of 7 representative antibiotics with lower limits of detection (LOD) by several hundred fold. Accurate results with absolute quantitation of the antibiotic in milk samples have been demonstrated using our approach integrated with a stable isotope-labelled analyte as the internal standard. Intrabacterial and extrabacterial antibiotic residues can be sensitively detected using our method. For the first time, we have revealed the mechanism of molecular deprotonation assisted by a CuCoO-GO matrix for its high performance in MALDI-TOF MS with synchrotron radiation techniques. This work provides a sensitive and robust matrix enabling MALDITOF MS applications in small molecule analysis, but also offers a distinctly important perspective on the molecular mechanism behind the material functions.

\section{Experimental}

\section{Chemicals}

All of the chemicals were purchased from Sigma Aldrich, Aladdin Industrial Corporation, or Shanghai Macklin Biochemical Co., Ltd. Sulfapyridine $-{ }^{13} \mathrm{C}$ was bought from WITEGA Laboratorien Berlin-Adlershof Gmbh (Germany). The milk samples were purchased from a local grocery store. Deionized water $\left(18 \mathrm{M} \Omega \mathrm{cm}^{-1}\right)$ was used in the experiments.

\section{Preparation of CuCoO-GO}

A modified Hummers' method was used to prepare graphene oxide (GO) from natural graphite. A GO solution was prepared by re-suspending $80 \mathrm{mg}$ GO in $80 \mathrm{~mL}$ isopropanol, followed by sonication for $20 \mathrm{~min} .38 .3 \mathrm{mg}(0.192 \mathrm{mmol}) \mathrm{Cu}\left(\mathrm{CH}_{3} \mathrm{COO}\right)_{2} \cdot \mathrm{H}_{2} \mathrm{O}$ and $12 \mathrm{mg}(0.048 \mathrm{mmol}) \mathrm{Co}\left(\mathrm{CH}_{3} \mathrm{COO}\right)_{2} \cdot 4 \mathrm{H}_{2} \mathrm{O}$ were mixed with the GO isopropanol solution. The mixture was heated to $80^{\circ} \mathrm{C}$ for $1 \mathrm{~h}$ with a reflux set-up. After $80 \mathrm{~mL}$ of deionized water was added into the reaction, the mixture was kept at $80{ }^{\circ} \mathrm{C}$ for $30 \mathrm{~min}$. The product was cooled to room temperature, then collected by centrifugation at $8000 \mathrm{rpm}$ for $3 \mathrm{~min}$ with an ethanol rinse. This was dried in an oven at $70{ }^{\circ} \mathrm{C}$ for $12 \mathrm{~h}$. The samples of CuCoO-GO with different $\mathrm{Cu} / \mathrm{Co}$ atom ratios (including $\mathrm{CuO}-\mathrm{GO}$ ) were prepared similarly by referring to this procedure. ${ }^{37}$

\section{Characterization}

High-resolution transmission electron microscopy (HRTEM) images were acquired with a FEI Tecnai G2 F20 S-TWIN TEM. Atomic force microscopy (AFM) images were obtained by using a Veeco Multimode $\mathrm{V}$ atomic force microscope. Raman spectra were collected using a HR 800 Raman spectroscope (J Y, France). The Fourier Transform Infrared (FT-IR) spectrum was obtained using a Bruker Fourier Transform Infrared Spectrometer (Hyperion). X-ray photoelectron spectroscopy (XPS) characterization was performed with a KRATOS Axis ultra DLD X-ray photoelectron spectroscope. X-ray diffraction (XRD) data were collected by a PANalytical B.V. Empyrean powder diffractometer equipped with a PIXcel3D detector. Scanning electron microscope (SEM) images of samples were acquired by an S-4700 SEM (Hitachi, Japan).

\section{Sample preparation for MALDI-TOF MS analysis}

The conventional organic matrices of $\alpha$-cyano-4hydroxycinnamic acid (CHCA) and 3,5-dimethoxy 4-hydroxycinnamic acid (SA) were individually prepared by a standard method. ${ }^{38,39}$ Graphene oxide, CuO-GO, CuO, CuCoO, CuCoOGO were all dispersed in water at a concentration of $1 \mathrm{mg} \mathrm{mL}{ }^{-1}$. The analytes of 7 different antibiotics and 3 additional chemical analogs, including sulfadiazine (SDZ), sulfamethoxazole (SMX), norfloxacin, ampicillin, benzylpenicillin, streptomycin, tetracycline, sulfapyridine, sulfonamide and pyrimethanil, were dissolved in water or ethyl alcohol as the standard mother solutions. They were subsequently diluted to a series of different concentrations as specified in the experiments. The sample spots on the MALDI plate were prepared by a standard method of sandwiching the matrix and analyte solutions. The liquid volume in each pipetting step for one sample spot was 1 $\mu \mathrm{L}$. The milk samples were mixed with different concentrations of the antibiotic solutions for the tests. They were defatted by mixing with $20 \%$ TFA and citrate buffer $(0.4 \mathrm{M}, \mathrm{pH}=4)$, followed by centrifugation ( $4000 \mathrm{rpm}$ for $20 \mathrm{~min}$ ) to collect the supernatants for MALDI-TOF MS analysis. ${ }^{40}$

\section{Mass Spectrometry}

The MALDI-TOF MS data were acquired using a MALDI TOF/ TOF Ultraflextreme mass spectrometer equipped with a SmartBeam II Nd:YAG/355 nm laser operating at $2000 \mathrm{kHz}$ (Bruker Daltonics, Inc., Billerica, MA), either in the reflective positiveion or negative-ion mode. The laser energy was optimized at $30 \%$ corresponding to $57 \mu \mathrm{J}$ per pulse (laser pulse duration: 3 ns). The laser spot size was approximately 50-100 $\mu \mathrm{m}$. Each sample was tested with a repetition of $n=4$. An accumulation of 3000 laser shots was applied for each spectrum.

For the comparison of different matrices, the negative ion mode was carried out using CuCoO-GO, GO, CuO-GO, and $\mathrm{CuCoO}$ as nano-matrices for detection of SDZ and SMX. In addition, the noise baselines of traditional matrices of CHCA and SA were also included in the measurements under identical instrumental conditions. 


\section{Antimicrobial susceptibility testing}

The minimal inhibitory concentrations (MICs) of sulfadiazine sodium (SDS) for E. coli and S. aureus were determined on the basis of microdilution assay. ${ }^{41}$ First, a single colony of $E$. coli and $S$. aureus on the solid Luria-Bertani (LB) agar plate was transferred into $5 \mathrm{~mL}$ of liquid LB culture medium and grown overnight at $37^{\circ} \mathrm{C}$. This preculture was diluted in $5 \mathrm{~mL}$ of liquid LB culture medium (dilution ratio: $1: 20$ ) and grown for $16 \mathrm{~h}$ at $37{ }^{\circ} \mathrm{C}$. The bacterial suspension was then diluted with LB culture medium to $1 \times 10^{6} \mathrm{CFU} \mathrm{mL}^{-1}$ (colony-forming unit per milliliter). Different concentrations of sulfadiazine sodium from 2 to $0.125 \mathrm{mg} \mathrm{mL}^{-1}$ were mixed with an equal volume of the diluted bacterial suspension and incubated at $37^{\circ} \mathrm{C}$ for $20 \mathrm{~h}$. The tube without sulfadiazine sodium was included as a blank control. Finally, the optical density values at $600 \mathrm{~nm}\left(\mathrm{OD}_{600}\right)$ of the solutions were recorded by a Varioskan Flash (Thermo Scientific Company, USA).

In parallel, sample aliquots of the bacteria incubated with antibiotics were centrifuged at $5000 \mathrm{rpm}$ for 5 minutes. The supernatant solutions were collected for analysis by $\mathrm{CuCoO}-\mathrm{GO}$ assisted MALDI MS. Simultaneously, the pellets of bacteria were resuspended in a Tris-HCL buffer and centrifuged again. After the washing steps, the bacteria were lysed by sonication in equal volume aliquots. The lysed bacteria were also tested by $\mathrm{CuCoO}-$ GO assisted MALDI MS.

\section{Synchrotron radiation experiments}

X-ray absorption spectroscopy (XAS) experiments were performed at the Shanghai Synchrotron Radiation Facility (SSRF, $14 \mathrm{~W}$ and 08U), the Beijing Synchrotron Radiation Facility (BSRF, soft X-ray beamline), and the National Synchrotron Radiation Laboratory (NSRL, XMCD beamline). The in situ XAS experiments at the $\mathrm{Cu}$ K-edge were performed at Beamline $14 \mathrm{~W}$ at the SSRF, in which the samples of CuCoO-GO with or without SDZ was pressed on the $3 \mathrm{M}$ tape with the option of UV irradiation for measurements.

\section{Results and discussion}

\section{Synthesis and characterization of CuCoO-GO}

CuCoO-GO was synthesized by the method of a refluxing reaction using $\mathrm{Cu}\left(\mathrm{CH}_{3} \mathrm{COO}\right)_{2} \cdot \mathrm{H}_{2} \mathrm{O}$ and $\mathrm{Co}\left(\mathrm{CH}_{3} \mathrm{COO}\right)_{2} \cdot 4 \mathrm{H}_{2} \mathrm{O}$ as the metal precursors in a mixture with the $\mathrm{GO} /$ isopropanol suspension. ${ }^{37}$ As shown in Fig. 1a, the TEM image indicates that the as-prepared nanocomposites are few-layered GO nanosheets decorated with nanoparticles with an average size of $10 \mathrm{~nm}$. The elemental mapping of a localized area by random selection further reveals the distribution of $\mathrm{O}, \mathrm{Cu}$, Co atoms in the nanocomposites. The AFM image suggests that $\mathrm{CuCoO}-\mathrm{GO}$ nanocomposites are uniform with a typical lateral dimension of several hundred nanometers (Fig. 1b). The height profiling by AFM demonstrates thicknesses of 1-2 nm, suggesting that the nanocomposites contain only a couple of GO layers (Fig. 1(b1 and b2)). In the Raman spectral analysis of $\mathrm{CuCoO}-\mathrm{GO}, \mathrm{CuO}-$ $\mathrm{GO}$, and GO (the latter two as the control samples), there are two characteristic peaks at $1350 \mathrm{~cm}^{-1}$ (D-band) and $1580 \mathrm{~cm}^{-1}$ (G- band), which are respectively attributed to the vibration of $\mathrm{sp}^{3}$-bonded or $\mathrm{sp}^{2}$-bonded carbon atoms in the graphitic nanostructures (Fig. 1c). The appearance of a third peak at $2800 \mathrm{~cm}^{-1}$ (2D-band) in all of these three different samples suggests that the few-layered feature in the nanosheets is maintained for the CuCoO-GO nanocomposites after the synthetic procedure (Fig. 1c). The analysis by XRD also identifies the characteristic diffraction peaks contributed by GO in all of these three samples as specified (Fig. 1d). XPS spectral analysis of $\mathrm{CuCoO}-\mathrm{GO}$ verifies the elements copper, cobalt, carbon, and oxygen in the composition of the hybrid nanomaterials (Fig. S1a $\dagger$ ). In the Fourier Transform Infrared (FTIR) spectrum of CuCoO-GO, the peaks at $3400 \mathrm{~cm}^{-1}$ and $1570 \mathrm{~cm}^{-1}$ are attributed to the chemical groups $\mathrm{O}-\mathrm{H}$ and $\mathrm{C}=\mathrm{O}$ (stretching mode). The peak at $1250 \mathrm{~cm}^{-1}$ is attributed to the epoxy groups (Fig. S1b $\dagger$ ). In the contact angle measurements, CuCoO-GO $(\theta=$ $\left.5.1^{\circ}\right)$ exhibits a slightly enhanced hydrophilicity compared to GO $\left(\theta=20.8^{\circ}\right)$. This facilitates forming a relatively uniform film of nanoparticles on the substrate, which is favorable for the deposition of soluble analytes (Fig. S2 $\dagger$ ).

\section{The performance of the CuCoO-GO matrix for MALDI-TOF}

We compared the background baseline of the CuCoO-GO matrix without any analytes in MALDI-TOF against traditional organic matrices such as CHCA and SA under identical instrumental conditions. As shown in Fig. 2a, the organic matrices (CHCA or SA) produce intensive background noise in a low molecular weight range $(\mathrm{m} / \mathrm{z}<700)$. In contrast, the baseline of CuCoO-GO is ultraclean, at least 4 orders of magnitude lower than the traditional organic matrices in noise level. Its property of being free from the interference of fragmented species suggests a good potential for CuCoO-GO as a MALDI matrix to detect small molecules in the range of $m / z<700$. The background baselines of the pristine nanomaterials of $\mathrm{GO}$ and $\mathrm{CuO}-$ GO are included in the ESI (Fig. S3 $\dagger$ ) for reference.

The performance of the CuCoO-GO matrix was evaluated by MALDI-TOF MS in both the positive-ion and negative-ion modes for the detection of small molecular antibiotics, including sulfadiazine (SDZ) and sulfamethoxazole (SMX). As shown in Fig. 2b, there are clear characteristic peaks for the analytes without the interference of background noise in both of these two modes. In the positive-ion mode, two characteristic peaks are attributed to $\mathrm{SDZ}\left(\mathrm{m} / \mathrm{z} 273[\mathrm{M}+\mathrm{Na}]^{+}, 289[\mathrm{M}+\mathrm{K}]^{+}\right)$or SMX $\left(\mathrm{m} / z 276[\mathrm{M}+\mathrm{Na}]^{+}, 292[\mathrm{M}+\mathrm{K}]^{+}\right)$. In the negative-ion mode, each of the small molecule analytes are identified with the characteristic peak of the deprotonated ion $[\mathrm{M}-\mathrm{H}]^{-}(\mathrm{m} / \mathrm{z} 249[\mathrm{M}-$ $\mathrm{H}]^{-}$for SDZ; $m / z 252[\mathrm{M}-\mathrm{H}]^{-}$for SMX). All of these characteristic peaks, featured with a high signal-to-noise ratio, benefit from the high ionization efficiency and low-background noise of the CuCoO-GO matrix. The reproducibility of the CuCoO-GO matrix in MALDI MS has been evaluated by two different modes, including tests of different positions in one spot and tests of different spots in parallel from the same sample. The relatively low coefficient of variation $(\mathrm{CV}, \sim 5 \%)$ in both of these two modes indicates a highly reproducible performance for the 

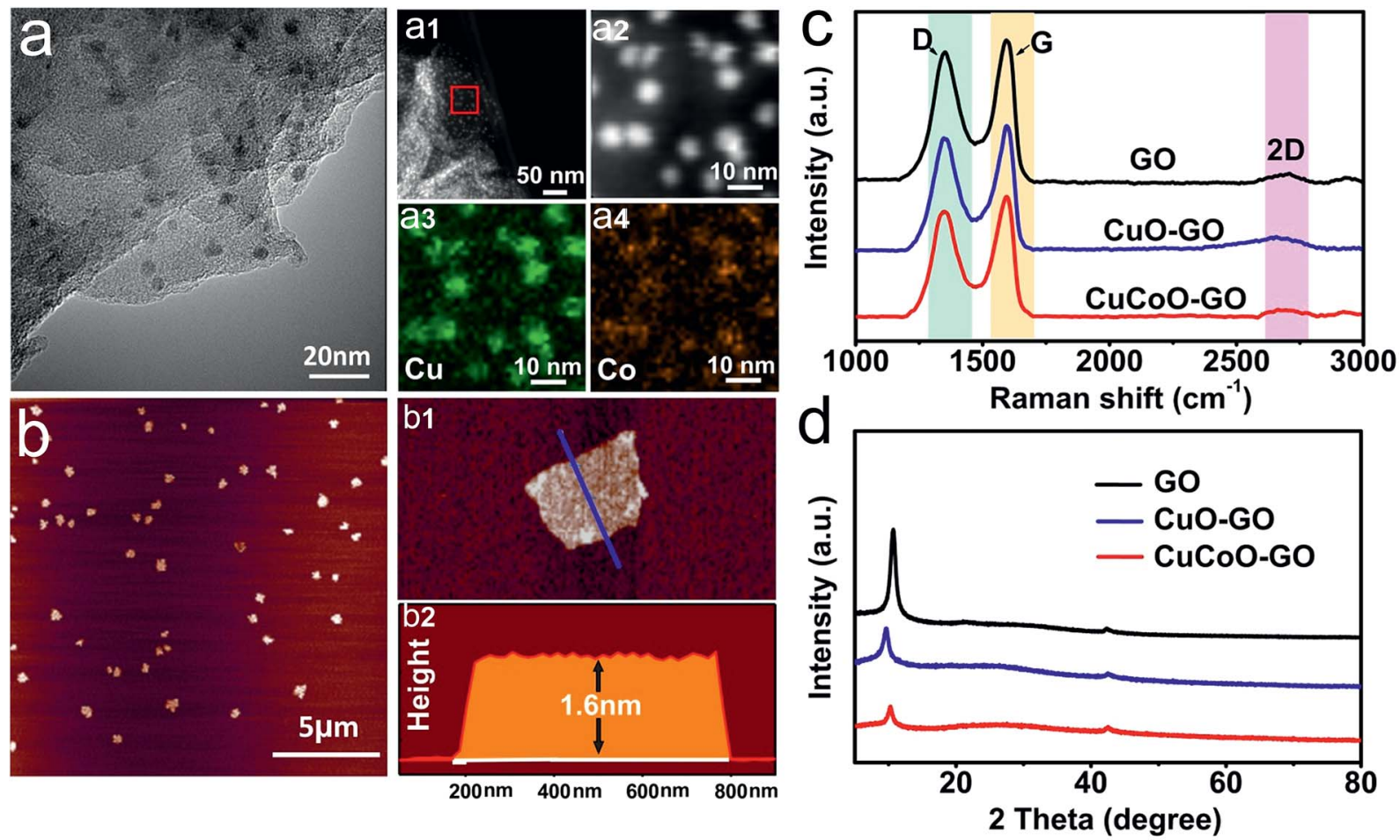

Fig. 1 Characterization of $\mathrm{CuCoO}-\mathrm{GO}(\mathrm{CuO}-\mathrm{GO}$ and $\mathrm{GO}$ as the control samples). (a) TEM image; (a1) dark-field TEM image; (a2) zoomed-in view of the dark-field in the red box; (a3 and a4) corresponding elemental mapping in the red box: Cu (green) and Co (brown). Sample: CuCoO$\mathrm{GO}$; scale bars as specified. (b) AFM image of $\mathrm{CuCoO}-\mathrm{Go}$ in the larger field of view; (b1) AFM of a single nanosheet of CuCoO-Go; (b2) height profile along the corresponding blue line of (b1). Scale bars: $5 \mu \mathrm{m}$. (c and d) Raman spectra and X-ray diffraction (XRD) patterns of GO, CuO-GO and $\mathrm{CuCOO}-\mathrm{GO}$.

CuCoO-GO matrix in the ionization of small molecular antibiotics for detection (Fig. $\mathrm{S} 4 \dagger$ ).

We performed quantitative analysis on SDZ by including an isotope labelled analog of the analyte with reference to the standard method. Stable isotope labelled sulfapyridine- ${ }^{13} \mathrm{C}$ was selected as the internal standard due to its great similarity in chemical structure to SDZ. It was further validated for being a good choice by the linear calibration curves in the titration of SDZ concentrations in MALDI MS. As shown in Fig. 2c, the characteristic peaks for SDZ $(\mathrm{m} / \mathrm{z} 249)$ and sulfapyridine $-{ }^{13} \mathrm{C}(\mathrm{m} /$ $z$ 254) are presented in the mass spectra. The intensities of these peaks are consistent with their spiking ratios in the water or the milk samples. There is excellent linearity for the calibration curve in the aqueous solution prepared with either water $\left(R^{2}=\right.$ $0.983)$ or milk $\left(R^{2}=0.982\right)$ between the MS signal intensity ratios (SDZ: sulfapyridine $-{ }^{13} \mathrm{C}$ ) and the concentrations of SDZ (10-1000 $\left.\mu \mathrm{g} \mathrm{L}^{-1}\right)$. In addition, the MS spectrum of the blank milk without any antibiotic addition is available in the ESI (Fig. S5 ${ }^{\dagger}$ ) as the negative control. It verifies that the characteristic peaks of the antibiotic analytes are not contaminated. These results provide good evidence to demonstrate the usefulness of our approach with the CuCoO-GO matrix for residual antibiotic quantification in milk samples.

The limit of detection (LOD) using our approach was determined by titrating the analytes and linear extrapolation of the signal-to-noise ratio $(\mathrm{S} / \mathrm{N}=3)$, according to the standard method. ${ }^{42}$ As previously demonstrated in Fig. 2, the CuCoO-GO matrix is featured with a clean baseline in MALDI MS, which is important for improved LOD in the detection of antibiotics. The detailed values of LOD are summarized in Table S1 and Fig. S6 in the ESI, $\uparrow$ including SDZ, SMX, and the other five representative antibiotics. For instance, the LOD for SDZ antibiotic is 0.5 $\mu \mathrm{g} \mathrm{L}^{-1}$ by our approach, which is nearly 500 fold lower than the previously reported results in the literature. This value is also far lower than the maximum residue limit (MRL, $250 \mu \mathrm{g} \mathrm{L}^{-1}$ for SDZ) in the milk regulated by the China National Standards. The superior performance of the CuCoO-GO matrix was validated by testing the other antibiotics in the list (Table S1 $\dagger$ ), and comparing with the results using different matrices (including graphene, graphene oxide, and other common matrices) in the literature. ${ }^{\mathbf{1 5 , 4 0 , 4 3 - 4 5}}$ This suggests that our approach of using the CuCoO-GO matrix can sufficiently meet the technical requirement for the sensitive detection of antibiotics.

Antibiotic susceptibility testing (AST) has been widely used in clinical microbiology labs to determine sensitiveness or the resistance of individual bacterial isolates to any specific antimicrobial agents. The methods for AST include broth dilution test, antimicrobial gradient method, disk diffusion test, or AST stripes, which typically rely on optical measurements or empirical naked-eye observations. ${ }^{41}$ Recently an important advance of $\mathrm{TiO}_{2}$-assisted MALDI TOF MS was developed to detect antimicrobial resistance-relevant proteins while minimizing sample preparation steps, which promotes simultaneous species identification and antimicrobial resistance 

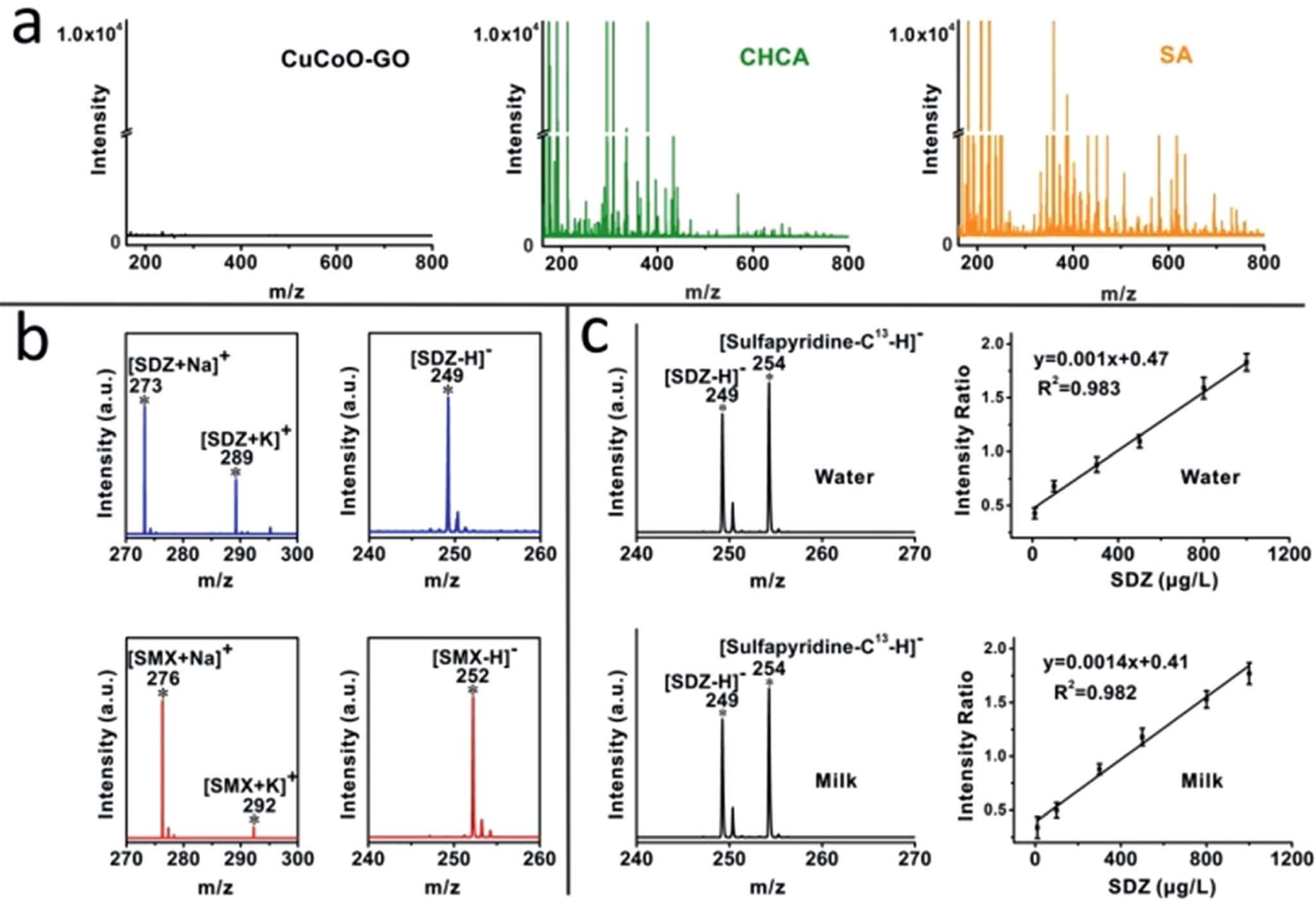

Fig. 2 High performance of the CuCoO-GO matrix in MALDI mass spectrometry for antibiotic analysis. (a) The background noise of the CuCoO-GO matrix in comparison with traditional organic matrices of CHCA and SA, in the negative-ion mode. (b) The mass spectra of SDZ and SMX in the positive-ion and negative-ion modes using the CuCoO-GO matrix. (c) Mass spectra of SDZ and sulfapyridine- ${ }^{13} \mathrm{C}$ (internal standard) in water solution or the milk sample, and the calibration curves for absolute quantitation of SDZ in the negative-ion mode $(n=4)$. Error bars: standard deviation.

analysis. ${ }^{46}$ Inspired by this work, we attempted to explore the utility of the $\mathrm{CuCoO}-\mathrm{GO}$ matrix for the application of antimicrobial resistance analysis by measuring both the intrabacterial and extrabacterial antibiotic residues (Fig. 3a). We performed AST experiments to test the antibiotic SDS on E. coli and $S$. aureus, using a standard microdilution assay (Fig. S7†). The morphological changes of E. coli and S. aureus induced by the antibiotic treatments were characterized by scanning electron microscopy (SEM, Fig. S8 $\dagger$ ). The bacterial samples treated with different concentrations of SDS were centrifuged to separate the supernatants and bacterial pellets, which were subsequently processed to analyze the antibiotic residues inside or outside the bacteria by $\mathrm{CuCoO}-\mathrm{GO}$ assisted MALDI MS. As shown in Fig. $3 \mathrm{~b}$ and c, in the mass spectra of E. coli and $S$. aureus lysates, the characteristic peaks of SDS $(\mathrm{m} / \mathrm{z} 273)$, sulfapyridine $-{ }^{13} \mathrm{C}(\mathrm{m} / \mathrm{z}$ 278 ), and a potential metabolite (4-OH-SDS, $\mathrm{m} / \mathrm{z} 289$ ) can be clearly identified. The calibration curve in the plot (Fig. 3d) according to the standard isotope internal control method exhibits a good linearity $\left(R^{2}=0.982\right)$, indicating a reliable performance of our approach for the antibiotic quantification using the samples of bacterial cultivation. As shown in Fig. 3e and f, the residual SDS outside and inside the E. coli or S. aureus after antibiotic treatment can be quantitatively analyzed. It suggests a trend that the proportion of SDS penetrating and leftover inside the bacteria increases, driven by the increase of the initial antibiotic dosages. Aligned to the standard AST experiments, our method can provide more detailed information on the uptake and potential metabolic changes of antibiotics, which are essential for the understanding of bacterial resistance mechanisms. ${ }^{28,47}$

Our design of hybridizing $\mathrm{CuCoO}$ nanoparticles on GO nanosheets is critical for the development of a high performance matrix. Indeed, GO plays an important role in this hybrid nanomaterial. GO nanosheets contain aromatic structures and chemical groups such as hydroxyl, epoxy, and carboxyl groups, enhancing molecular interactions with analytes and serving as a matrix choice. ${ }^{32,48}$ We designed experiments to compare the performance of $\mathrm{CuO}$ or $\mathrm{CuCoO}$ nanoparticles without $\mathrm{GO}$, and the CuCoO-GO hybrid matrix in the detection of SDZ and SMX molecules. In contrast to the high efficiency of $\mathrm{CuCoO}-\mathrm{GO}$ in the ionization of small molecules, $\mathrm{CuO}$ or $\mathrm{CuCoO}$ nanoparticles as the matrix can only produce very low signals for the detection of SDZ or SMZ. The difference in the signal intensity is nearly two orders of magnitude (Fig. S9†). This suggests that bare $\mathrm{CuO}$ 
a

.

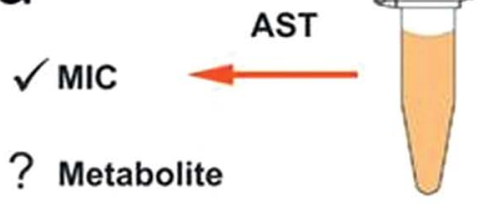

? Inside/Outside bacteria
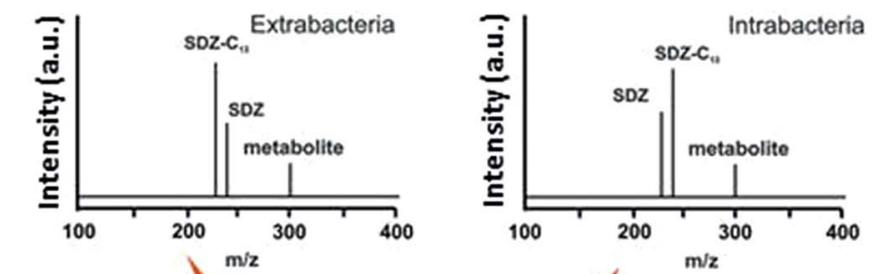

MS

- Antibioic Metabolite
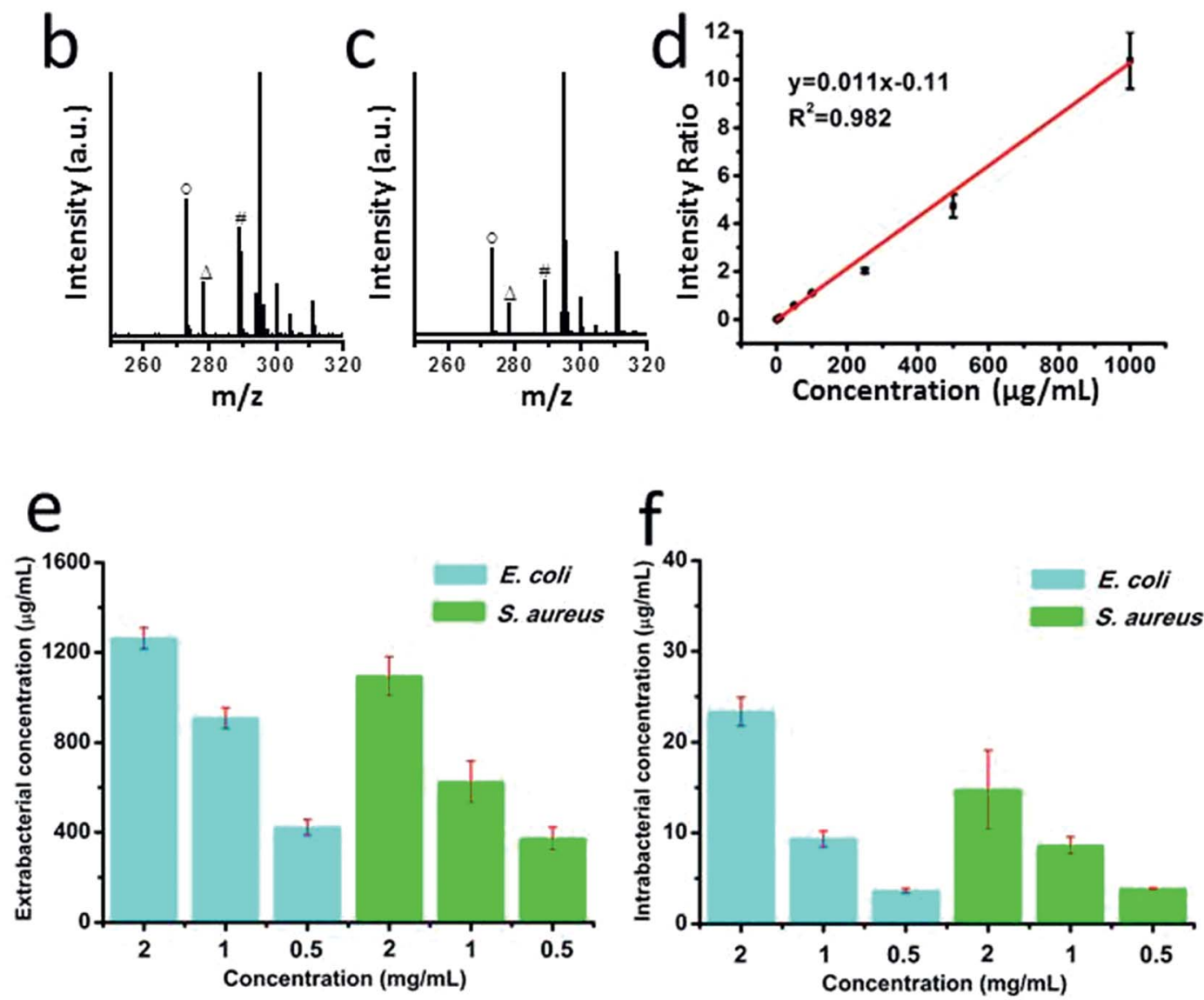

Fig. 3 (a) Schematic diagram of antibiotic detection comparing our method to standard antibiotic susceptibility testing (AST). (b) Mass spectrometry data of $E$. coli lysates. (c) Mass spectrometry data of $S$. aureus lysates. $O, \Delta$ and \# represent peaks of SDS, sulfapyridine- ${ }^{13} \mathrm{C}$ and a potential SDS metabolite in (b) and (c). (d) The calibration curves for absolute quantitation of sulfadiazine sodium using sulfapyridine- ${ }^{13} \mathrm{C}$ as the internal standard $(n=4)$. (e) Extrabacterial and (f) intrabacterial SDS residue detected by our method, after the treatment of bacteria for $20 \mathrm{~h}$ with different antibiotic dosages: $2 \mathrm{mg} \mathrm{mL}^{-1}, 1 \mathrm{mg} \mathrm{mL}^{-1}, 0.5 \mathrm{mg} \mathrm{mL}^{-1}$ light blue: E. coli; green: $S$. aureus. Data points were collected from 2 separate batches. Each of the batches contained a repetition of 4 in parallel. Error bar: standard deviation.

or $\mathrm{CuCoO}$ nanoparticles without GO as the matrix are limited when promoting small molecule ionization. In addition, we compared the efficiency in laser desorption ionization (LDI) of the analytes using different types of graphene oxide-based matrices including $\mathrm{GO}, \mathrm{CuO}-\mathrm{GO}, \mathrm{CuCoO}-\mathrm{GO}$ under identical conditions. As shown in Fig. 4, both SDZ and SMX can be detected by using any of the above matrices. However, there appears to be prominent differences among these GO-based 
matrices in terms of the signal-to-noise $(\mathrm{S} / \mathrm{N})$ ratios for the detection of the antibiotic analytes. The CuCoO-GO matrix allows for the detection of either SDZ or SMX with the highest S/ $\mathrm{N}$ ratio, which is approximately 2 fold higher than the $\mathrm{CuO}-\mathrm{GO}$ matrix, or 8 fold higher than the bare GO matrix. The significantly enhanced $\mathrm{S} / \mathrm{N}$ ratio by the $\mathrm{CuCoO}-\mathrm{GO}$ matrix suggests a synergistic effect on LDI of the analytes, contributed to by the hybrid nanosystem of metal oxides and GO.

\section{Mechanistic studies by synchrotron radiation-based techniques}

Synchrotron radiation-based X-ray absorption spectroscopy (XAS) has been widely used to reveal the chemical state information of elements in various materials..$^{37,49,50}$ Here we employed this technique to explore the absorption changes of the specific elements in the CuCoO-GO matrix with/without small molecule analytes for mechanistic studies. The C K-edge XAS spectra (Fig. S10 $\dagger$ ) validate the interactions between SDZ molecules and CuCoO-GO nanosheets accompanying adsorption. The pristine sample of CuCoO-GO exhibits a XAS spectrum similar to typical GO-based materials as reported in the literature. ${ }^{37}$ The sample CuCoO-GO after addition of SDZ presents clear signals for its adsorption with the evidence the shifted peak A and the intensity increase in peak B. After the addition of the analyte (SDZ), there is no significant shift in the absorption peak of the $\mathrm{Cu}$ L-edge in CuO-GO (black, red curves in Fig. 5a and b). In contrast, the absorption peak of the $\mathrm{Cu}$ L-edge in CuCoO-GO is shifted to a higher energy level
$(\Delta E \approx 0.3 \mathrm{eV})$, suggesting a change for the higher chemical state of the $\mathrm{Cu}$ element (blue, purple curves in Fig. 5a and b). This change can be attributed to the interactions between SDZ and the $\mathrm{Cu}$ element, which induce an effect of partial electron shift from $\mathrm{Cu}$ to $\mathrm{O}$ in SDZ. Compared to $\mathrm{CuO}-\mathrm{GO}, \mathrm{CuCoO}-\mathrm{GO}$ with Co doping may facilitate a delocalized electron effect by promoting interactions between $\mathrm{CuCoO}$ and GO. Furthermore, in the in situ Cu K-edge XAS experiments (Fig. 5c and d and $\mathrm{S} 11 \dagger$ ), addition of SDZ results in an intensity increase for the absorption peak of CuCoO-GO, corresponding to a chemical state change of $\mathrm{Cu}$ due to a partial electron shift to atoms with higher electronegativity such as the oxygen atom. These results are consistent with the previous measurements of $\mathrm{Cu}$ L-edge absorption in Fig. 5a and b. Interestingly, there is an inverse change for the absorption peak intensity at the $\mathrm{Cu}$ K-edge when the sample mixture of CuCoO-GO and SDZ is illuminatedin situ by UV light. This suggests recovery of the chemical state of copper, accompanying the removal of SDZ and a drop in the interactions between SDZ and $\mathrm{Cu}$ due to UV irradiation.

We propose a mechanism for the ionization of small molecules with the CuCoO-GO matrix, based on the above experimental evidence collectively (Fig. 6). The hybrid nanomaterial CuCoO-GO is able to ionize the small molecular analyte (SDZ) with high efficiency assisted by clustered $\mathrm{Cu}, \mathrm{Co}, \mathrm{O}$ atoms on the GO nanosheets. SDZ molecules can be adsorbed on the surface of CuCoO-GO by hydrogen bonds and van der Waals forces. The $\mathrm{Cu}$ and $\mathrm{O}$ sites on the matrix can respectively interact with the $\mathrm{S}=\mathrm{O}$ bond and the $-\mathrm{NH}-$ group in the SDZ molecule. The
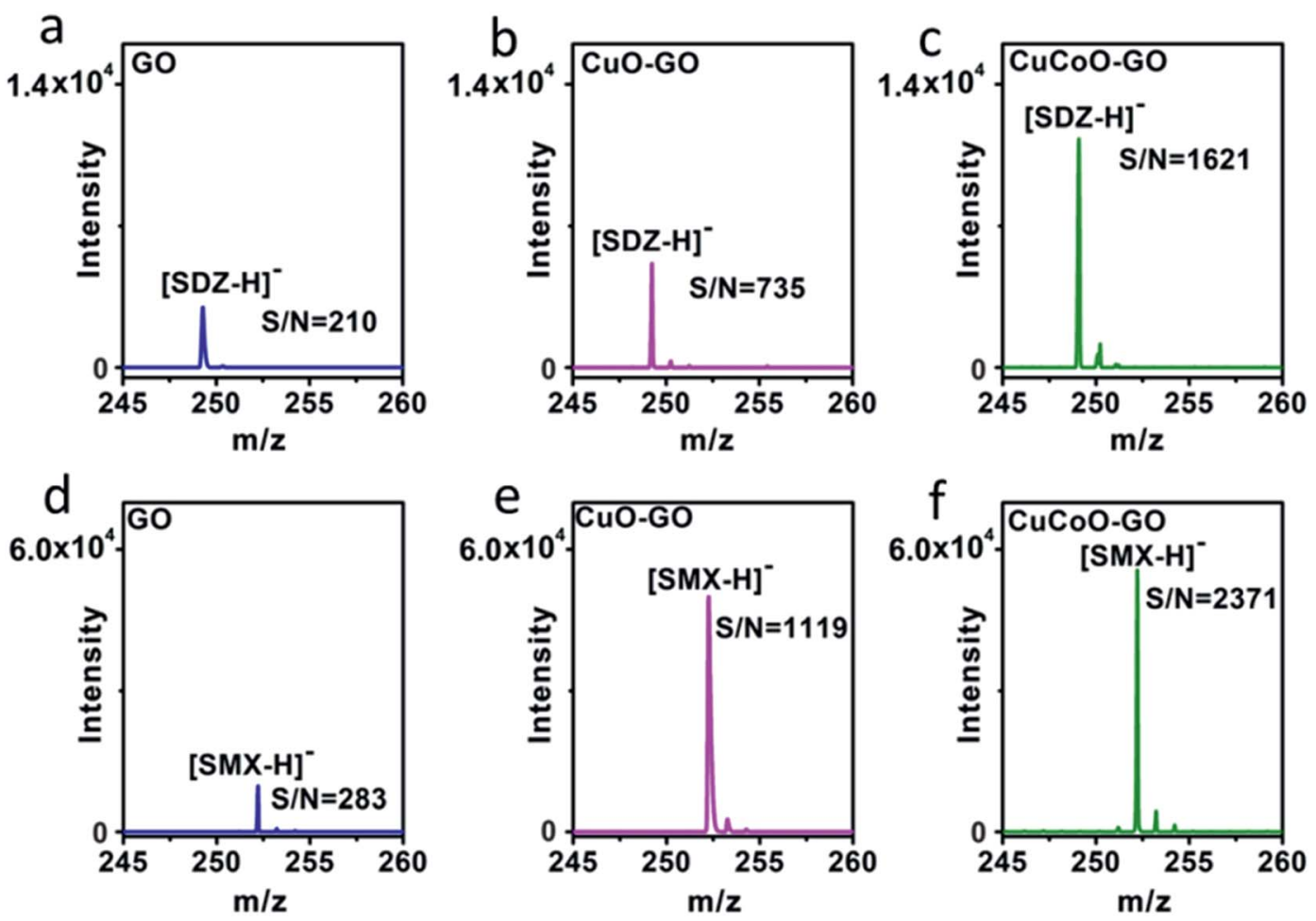

Fig. 4 Comparison of performance of GO, CuO-GO and CuCoO-GO matrices in the MALDI-TOF MS detection of antibiotics. (a-c) SDZ; (d-f) $\mathrm{SMX}$, under identical conditions (in the negative-ion mode, accumulated to 3000 shots, concentration: SDZ with $0.05 \mu \mathrm{g} \mathrm{mL}{ }^{-1}, \mathrm{SMX}$ with $1 \mu \mathrm{g}$ $\left.\mathrm{mL}^{-1}\right)$. 

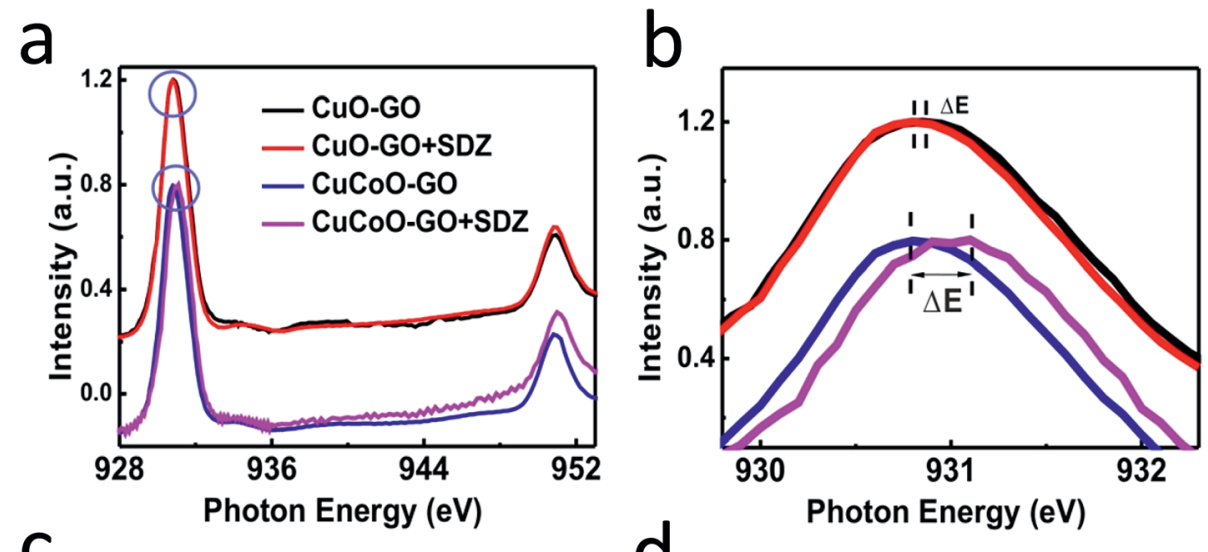

C

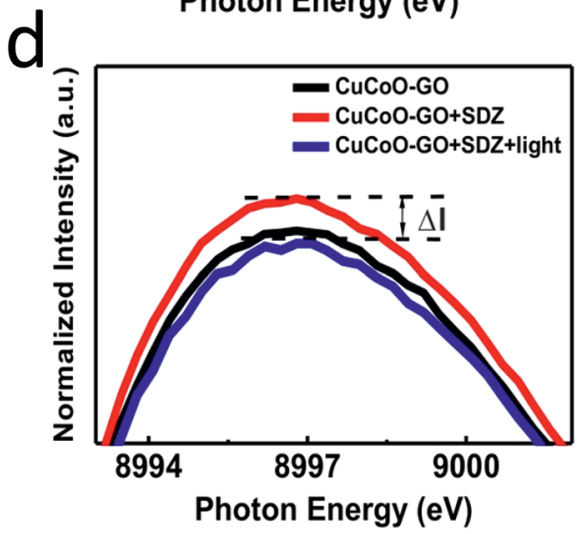

Fig. 5 Investigation on the interactions between SDZ and CuCoO-GO by synchrotron radiation-based techniques. (a) Hard X-ray XAS spectra of CuO-GO and CuCOO-GO with and without addition of SDZ. (b) Zoomed-in view of the peaks highlighted in the blue circles of (a). (c) In situ hard X-ray XAS spectra of CuCoO-GO at the Cu K-edge (d) Zoomed-in view of the peaks highlighted in the blue circle of (c).

presence of Co as the doping element in the matrix enables enhanced interactions between the atom clusters and GO, which could promote charge transfer between the CuCoO-GO matrix and SDZ in the ionization process. The energy input by laser may facilitate the deprotonation of SDZ, allowing for the ionized small molecule (SDZ) to be detected by the mass analyzer in the MALTI-TOF MS instrument. The hybrid CuCoOGO nanomaterial tends to exhibit a high proton affinity due to the delocalized pi-electron system across the GO surface. Due to the synergistic effect of the $\mathrm{Co}$ and $\mathrm{Cu}$ bimetallic oxides on the GO nanosheets, this hybrid matrix turns out to improve the ionization efficiency of small molecules, thus promoting their MS signal-to-noise ratios in detection.

The proposed mechanism is suitable for the negative-ion mode in MALDI MS, by taking advantage of the enhanced interactions between small molecules and the $\mathrm{CuCoO}-\mathrm{GO}$

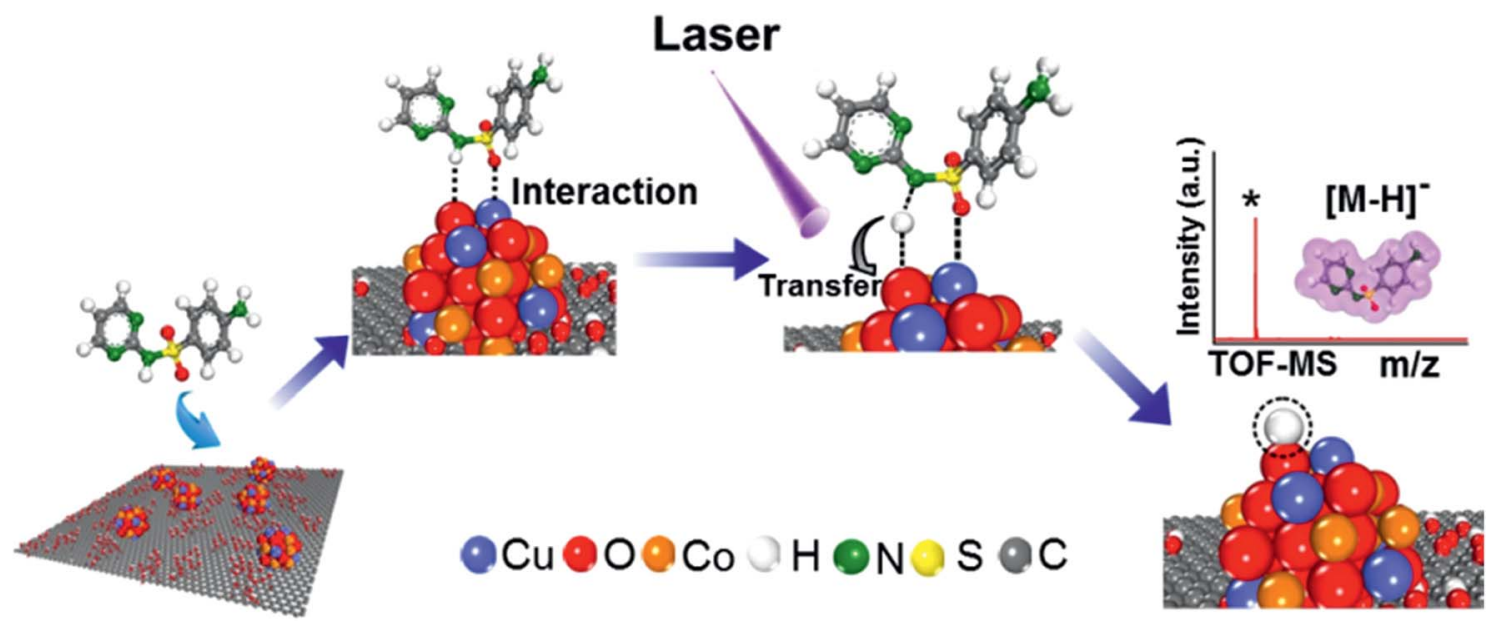

Fig. 6 Schematic diagram of the mechanism of SDZ ionization using the CuCoO-GO matrix for MALDI TOF mass spectrometry. 
matrix at the nano-interfaces. Distinctly different from the conventional matrices of organic molecules, the total atom number of the hybrid nanomaterial-based matrix is higher by several orders of magnitude. The nanomaterial-based matrix itself is resistant to desorption from the MALDI plate into the gas phase. Our experiments have also demonstrated that the CuCoO-GO matrix produces much fewer fragmented species (low-noise background, Fig. 2) in comparison to conventional matrices (CHCA, and SA) under irradiation with a UV laser. Therefore, the mechanism above mainly describes the dynamic interactions of matrix/small molecules at the condensed phasegas interfaces. However, ion reactions may also occur in the gas phase by complicated in-plume processes, which are important scenarios for examining the complete picture of MALDI ionization. ${ }^{51-53}$ There are several representative types of secondary ion reactions such as proton-transfer reactions, cationization reactions, electron-transfer reactions, and electron-capture reactions. ${ }^{\mathbf{5 1 , 5 2 , 5 4}}$

The gas-phase proton-transfer processes include matrixmatrix reactions and matrix-analyte reactions. ${ }^{49}$ As for matrixmatrix reactions,

$$
\begin{gathered}
\mathrm{M}^{+\bullet}+\mathrm{M} \rightarrow \mathrm{MH}^{+}+(\mathrm{M}-\mathrm{H})^{\bullet} \\
(\mathrm{M}-\mathrm{H})^{\bullet}+\mathrm{e}^{-} \rightarrow(\mathrm{M}-\mathrm{H})^{-} \\
\mathrm{M}+\mathrm{e}^{-} \rightarrow(\mathrm{M}-\mathrm{H})^{-}+\mathrm{H}^{\cdot}
\end{gathered}
$$

the matrix-matrix reaction (1) is dependent on the generation of radical cations from the matrix $\left(\mathrm{M}^{+}\right)$, which is likely to be in lower abundance for the $\mathrm{CuCoO}-\mathrm{GO}$ matrix than conventional organic matrices. Either the species $(\mathbf{M}-\mathbf{H})^{*}$ from reaction (1), which may subsequently capture a free electron (reaction (2)), or $\mathrm{M}$ in reaction (3) would lead to the generation of the negative ions $(\mathrm{M}-\mathrm{H})^{-}$. Relying on the existence of free electrons, the effect of these two potential processes may not be very pronounced because electrons tend to be extracted quickly by the large electric fields in MALDI TOF experiments. ${ }^{51}$

As for matrix-analyte reactions,

$$
\begin{gathered}
\mathrm{MH}^{+}+\mathrm{A} \rightarrow \mathrm{M}+\mathrm{AH}^{+} \\
(\mathrm{M}-\mathrm{H})^{-}+\mathrm{A} \rightarrow \mathrm{M}+(\mathrm{A}-\mathrm{H})^{-}
\end{gathered}
$$

reaction (4) is involved with the production of positive ions from the analyte molecules, which is beyond the major focus of our mechanism on the negative-ion mode. In addition, the mass spectra in the positive-ion mode using the CuCoO-GO matrix indicates that $\mathrm{Na}^{+} / \mathrm{K}^{+}$cationized analytes are predominantly observed, instead of protonated ones. This phenomenon is also different from conventional organic matrices which typically assist producing the protonated species in many cases. In reaction (5), the generation of $(\mathrm{A}-\mathrm{H})^{-}$may potentially enhance the desired signal in the mass spectra in the negative-ion mode. However, based on the discussion above, the negative ions ( $\mathrm{M}-$ $\mathrm{H}^{-}$in the gas phase from $\mathrm{CuCoO}-\mathrm{GO}$ are in low abundance, thus limiting the actual effect of the enhancement by this reaction.
As for the gas-phase cationization processes or the electrontransfer reactions such as:

$$
\mathrm{M}^{+\cdot}+\mathrm{A} \rightarrow \mathrm{M}+\mathrm{A}^{+\cdot}
$$

they mainly produce either alkali metal ion adducts or positive ions of the analytes (reaction (6)). Therefore, their contributions are not further included in our discussion, since the mechanistic interpretation of this manuscript is focused on the negative-ion mode. The electron-capture dissociation reaction (7) also suffers from the fast distinction of free electrons:

$$
\mathrm{A}+\mathrm{e}^{-} \rightarrow(\mathrm{A}-\mathrm{H})^{-}+\mathrm{H}^{\cdot}
$$

Herein we attempt to discuss several representative categories of secondary ion reactions. The actual processes may involve more complicated mechanisms of generating secondary ions. It is noteworthy that the distinctive properties of hybrid nanomaterials may foster mechanistic studies significantly different from conventional organic matrices. The current mechanism that we propose highlights the dynamic interactions between the hybrid nanomaterial and small molecules at the condensed phase-gas interfaces. A further detailed investigation on the mechanisms of primary and secondary ion generation will be necessary in the future.

In order to examine whether the proposed mechanism is limited by any particular chemical groups of the analytes, we have added three molecules, including sulfapyridine, sulfonamide, and pyrimethanil, in the experiments using the CuCoOGO matrix. Sulfapyridine can be viewed as a derivative of SDZ, with a slight difference in the number of nitrogen atoms. The chemical structure of sulfonamide is similar to SDZ, except for the removal of the nitrogen-containing heterocyclic ring structure. Pyrimethanil also contains some structural characteristics of SDZ, but without the $\mathrm{S}=\mathrm{O}$ bond. The mass spectra of these molecules in comparison with SDZ have been updated in the ESI (Fig. S12 $\dagger$ ). The spectral data support that these three types of molecules can be efficiently ionized in MALDI TOF MS by using the CuCoO-GO matrix. In addition, our new matrix has been demonstrated to assist the ionization of different categories of antibiotics with an enhanced limit of detection. Several representative antibiotics such as tetracycline and norfloxacin are featured with other functional groups, primarily including $\mathrm{C}=\mathrm{O}, \mathrm{H}_{2} \mathrm{~N}-\mathrm{C}=\mathrm{O}, \mathrm{HO}-\mathrm{C}=\mathrm{O}$, and $-\mathrm{OH}$, instead of $\mathrm{S}=\mathrm{O}$ and $-\mathrm{NH}-$. Based on the experimental results, we propose that the mechanism of molecular interactions may be adapted to a relatively wide range of small molecules with different chemical groups. To the best of our knowledge, this is the first time investigation of the nanomaterial-assisted ionization mechanism of small molecules using synchrotron radiationbased techniques.

\section{Conclusion}

In summary, we have developed the hybrid $\mathrm{CuCoO}-\mathrm{GO}$ as a new MALDI matrix for the sensitive detection of antibiotics, and revealed the molecule-nanomaterial interactions for the 
ionization mechanism. The $\mathrm{CuCoO}-\mathrm{GO}$ matrix allows for the detection of various antibiotics with LOD improvement of several hundreds of fold compared with the literature reports. We have validated high reproducibility of this approach, and demonstrated its performance in the quantification of sulfonamides in milk samples. Our approach can provide quantitative analysis of extrabacterial and intrabacterial concentrations of antibiotics as well as their potential metabolites, which are difficult to obtain by conventional AST assays. A panel of commonly-used antibiotics and several additional chemical analogs have been successfully detected with our method. We expect that the CuCoO-GO matrix shall be able to allow sensitive detection of a wide range of biomolecules containing functional groups such as carboxyl, amine, amide, carbonyl, sulfonic, and hydroxyl groups. By taking advantage of synchrotron radiation-based techniques, we have investigated the interactions between the molecule SDZ and the CuCoO-GO matrix for the ionization mechanism of SDZ with the assistance of CuCoO-GO under UV irradiation. The pivotal understanding of the matrix/molecule interactions and the ionization mechanism may create knowledge for matrix development and the ultrasensitive detection of small molecules.

\section{Conflicts of interest}

There are no conflicts to declare.

\section{Acknowledgements}

This work is supported by the Major State Basic Research Development Program (2013CB932702), and by the National Natural Science Foundation of China (21874096, 21575095); a project supported by the Collaborative Innovation Center of Suzhou Nano Science and Technology, and by the Joint International Research Laboratory of Carbon-Based Functional Materials and Devices; a 111 project; the Priority Academic Program Development of Jiangsu Higher Education Institutions (PAPD); J. L. is supported by the "1000 Youth Talents" plan of the Global Expert Recruitment Program.

\section{References}

1 S. Leclercq, F. M. Mian, A. M. Stanisz, L. B. Bindels, E. Cambier, H. Ben-Amram, O. Koren, P. Forsythe and J. Bienenstock, Nat. Commun., 2017, 8, 1-12.

2 J. M. Mitchell, M. W. Griffiths, S. A. McEwen, W. B. McNab and A. J. Yee, J. Food Prot., 1998, 61, 742-756.

3 X. Z. Li, P. Plesiat and H. Nikaido, Clin. Microbiol. Rev., 2015, 28, 337-418.

4 R. Laxminarayan, A. Duse, C. Wattal, A. K. M. Zaidi, H. F. L. Wertheim, N. Sumpradit, E. Vlieghe, G. L. Hara, I. M. Gould, H. Goossens, C. Greko, A. D. So, M. Bigdeli, G. Tomson, W. Woodhouse, E. Ombaka, A. Q. Peralta, F. N. Qamar, F. Mir, S. Kariuki, Z. A. Bhutta, A. Coates, R. Bergstrom, G. D. Wright, E. D. Brown and O. Cars, Lancet Infect. Dis., 2013, 13, 1057-1098.

5 J. M. C. Cernadas, Arch. Argent. Pediatr., 2017, 115, 306-307.
6 A. M. Saviano, F. L. Francisco and F. R. Lourenco, Talanta, 2014, 127, 225-229.

7 B. Shaikh and W. A. Moats, J. Chromatogr. A, 1993, 643, 369378.

8 M. Dubois, D. Fluchard, E. Sior and P. Delahaut, J. Chromatogr. B: Biomed. Sci. Appl., 2001, 753, 189-202.

9 B. Blanchaert, E. P. Jorge, P. Jankovics, E. Adams and A. Van Schepdael, Chromatographia, 2013, 76, 1505-1512.

10 I. K. Cigic and H. Prosen, Int. J. Mol. Sci., 2009, 10, 62-115.

11 I. Y. Goryacheva, S. De Saeger, I. S. Nesterenko, S. A. Eremin and C. Van Peteghem, Talanta, 2007, 72, 1230-1234.

12 H. Y. Zhang and S. O. Wang, J. Immunol. Methods, 2009, 350, 1-13.

13 S. Ahmed, J. Ning, G. Y. Cheng, I. Ahmad, J. Li, M. Y. Liu, W. Qu, M. Iqbal, M. A. B. Shabbir and Z. H. Yuan, Talanta, 2017, 166, 176-186.

14 B. F. Cravatt, G. M. Simon and J. R. Yates, Nature, 2007, 450, 991-1000.

15 Y. C. Ling, L. N. Lin and Y. T. Chen, Rapid Commun. Mass Spectrom., 1998, 12, 317-327.

16 L. S. Cai, L. F. Sheng, M. C. Xia, Z. P. Li, S. C. Zhang, X. R. Zhang and H. Y. Chen, J. Am. Soc. Mass Spectrom., 2017, 28, 399-408.

17 R. Shi, H. Li, E. H. Wu, L. P. Xiong, R. Lv, R. C. Guo, Y. Liu, G. Q. Xu, Z. H. Kang and J. Liu, Nanoscale, 2017, 9, 84108417.

18 J. J. Hu, F. Liu and H. X. Ju, Angew. Chem., Int. Ed., 2016, 55, 6667-6670.

19 D. Figeys, L. D. McBroom and M. F. Moran, Methods, 2001, 24, 230-239.

20 Y. M. Zhao, T. W. Muir, S. B. H. Kent, E. Tischer, J. M. Scardina and B. T. Chait, Proc. Natl. Acad. Sci. U. S. A., 1996, 93, 4020-4024.

21 S. A. Stopka, C. Rong, A. R. Korte, S. Yadavilli, J. Nazarian, T. T. Razunguzwa, N. J. Morris and A. Vertes, Angew. Chem., Int. Ed., 2016, 55, 4482-4486.

22 A. N. Krutchinsky and B. T. Chait, J. Am. Soc. Mass Spectrom., 2002, 13, 129-134.

23 T. W. Jaskolla, W. D. Lehmann and M. Karas, Proc. Natl. Acad. Sci. U. S. A., 2008, 105, 12200-12205.

24 R. Chen, S. M. Chen, C. Q. Xiong, X. L. Ding, C. C. Wu, H. C. Chang, S. X. Xiong and Z. X. Nie, J. Am. Soc. Mass Spectrom., 2012, 23, 1454-1460.

25 E. Gemperline, S. Rawson and L. J. Li, Anal. Chem., 2014, 86, 10030-10035.

26 W. T. Chen, C. K. Chiang, Y. W. Lin and H. T. Chang, J. Am. Soc. Mass Spectrom., 2010, 21, 864-867.

27 T. R. Northen, O. Yanes, M. T. Northen, D. Marrinucci, W. Uritboonthai, J. Apon, S. L. Golledge, A. Nordstrom and G. Siuzdak, Nature, 2007, 449, 1033.

28 L. Huang, J. J. Wan, X. Wei, Y. Liu, J. Y. Huang, X. M. Sun, R. Zhang, D. D. Gurav, V. Vedarethinam, Y. Li, R. P. Chen and K. Qian, Nat. Commun., 2017, 8, 222.

29 Y. Gholipour, S. L. Giudicessi, H. Nonami and R. ErraBalsells, Anal. Chem., 2010, 82, 5518-5526.

30 R. Shi, X. Dai, W. F. Li, F. Lu, Y. Liu, H. H. Qu, H. Li, Q. Y. Chen, H. Tian, E. H. Wu, Y. Wang, R. H. Zhou, 
S. T. Lee, Y. Lifshitz, Z. H. Kang and J. Liu, ACS Nano, 2017, 11, 9500-9513.

31 S. M. Chen, H. Z. Zheng, J. N. Wang, J. Hou, Q. He, H. H. Liu, C. Q. Xiong, X. L. Kong and Z. X. Nie, Anal. Chem., 2013, 85, 6646-6652.

32 Y. K. Kim, H. K. Na, S. J. Kwack, S. R. Ryoo, Y. Lee, S. Hong, S. Hong, Y. Jeong and D. H. Min, ACS Nano, 2011, 5, 45504561.

33 B. Gulbakan, E. Yasun, M. I. Shukoor, Z. Zhu, M. X. You, X. H. Tan, H. Sanchez, D. H. Powell, H. J. Dai and W. H. Tan, J. Am. Chem. Soc., 2010, 132, 17408-17410.

34 S. M. Chen, C. Q. Xiong, H. H. Liu, Q. Q. Wan, J. Hou, Q. He, A. Badu-Tawiah and Z. X. Nie, Nat. Nanotechnol., 2015, 10, 176-182.

35 M. S. Khan, M. L. Bhaisare, S. Pandey, A. Talib, S. M. Wu, S. K. Kailasa and H. F. Wu, Int. J. Mass Spectrom., 2015, 393, 25-33.

36 W. J. Lu, Y. Li, R. J. Li, S. M. Shuang, C. Dong and Z. W. Cai, ACS Appl. Mater. Interfaces, 2016, 8, 12976-12984.

37 K. Feng, J. Zhong, B. H. Zhao, H. Zhang, L. Xu, X. H. Sun and S. T. Lee, Angew. Chem., Int. Ed., 2016, 55, 11950-11954.

38 I. P. Smirnov, X. Zhu, T. Taylor, Y. Huang, P. Ross, I. A. Papayanopoulos, S. A. Martin and D. J. Pappin, Anal. Chem., 2004, 76, 2958-2965.

39 R. F. Gao, X. Chen, C. T. Chen, R. Shi, F. Z. Ouyang, J. Z. Yang, D. P. Sun and J. Liu, Cellulose, 2016, 23, 24752485.

40 J. Y. Liu, Y. Liu, M. X. Gao and X. M. Zhang, J. Am. Soc. Mass Spectrom., 2012, 23, 1424-1427.
41 J. H. Jorgensen and M. J. Ferraro, Clin. Infect. Dis., 2009, 49, 1749-1755.

42 J. I. Kim, J. M. Park, J. Y. Noh, S. J. Hwang, M. J. Kang and J. C. Pyun, Chemosphere, 2016, 143, 64-70.

43 V. Springer, J. Jacksen, P. Ek, A. G. Lista and A. Emmer, Chromatographia, 2015, 78, 285-290.

44 S. Notari, C. Mancone, M. Sergi, F. Gullotta, N. Bevilacqua, M. Tempestilli, R. Urso, F. N. Lauria, L. P. Pucillo, M. Tripodi and P. Ascenzi, IUBMB Life, 2010, 62, 387-393.

45 J. J. A. van Kampen, P. C. Burgers, R. de Groot and T. M. Luider, Anal. Chem., 2006, 78, 5403-5411.

46 Y. D. Zhu, N. Gasilova, M. Jovic, L. Qiao, B. H. Liu, L. T. Lovey, H. Pick and H. H. Girault, Chem. Sci., 2018, 9, 2212-2221.

47 R. T. Zhang, Q. Qin, B. H. Liu and L. Qiao, Anal. Chem., 2018, 90, 3863-3870.

48 Q. Liu, M. T. Cheng, J. Wang and G. B. Jiang, Chem.-Eur. J., 2015, 21, 5594-5599.

49 K. Ghanbari, A. S. Elahi and M. Ghoranneviss, J. X-Ray Sci. Technol., 2017, 25, 15-23.

50 L. Chen, Y. Mi, X. Wan, B. Yin, Z. Yuan, L. He and Q. Li, Appl. Ecol. Env. Res., 2017, 15, 335-341.

51 R. Zenobi and R. Knochenmuss, Mass Spectrom. Rev., 1998, 17, 337-366.

52 R. Knochenmuss and R. Zenobi, Chem. Rev., 2003, 103, 441452.

53 A. G. Harrison, Mass Spectrom. Rev., 1997, 16, 201-217.

54 H. Ehring, M. Karas and F. Hillenkamp, Org. Mass Spectrom., 1992, 27, 472-480. 